\title{
RESEARCH IN CONTEXT EFFECTS OF GRADED AFFECTIVE VALENCE VIDEOS ON RESULTS OF MEASUREMENTS OF IMPLICIT ATTITUDES TOWARDS RISKY DRIVING
}

\author{
Irina Plotka, Nina Blumenau, Dmitry Igonin, Zhanna Vinogradova \\ Baltic International Academy, Latvia \\ irinaplotka@inbox.lv,nina.blum@gmail.com, latenta@balticom.lv, artgrupalv@gmail.com
}

\begin{abstract}
The research aim is to study the context effects of graded affective valence videos on the results of measurements of implicit attitudes towards risky driving. The research is based on theoretical conceptualizations of explicit-implicit attitudes, discrepancy-consistency of their measurements presented in dual-process models of attitudes. Research questions: Have the results of measurements of implicit attitudes towards speeding using Implicit Association Test (IAT) and the results of measurements of explicit attitudes using self-reported procedures changed in response to context effects? What effect did the context factors have on results consistency of implicit and explicit measurements of attitudes towards speeding? Have the correlations between implicit and explicit attitudes towards speeding and personality traits changed in response to contextual effects? Participants: 66 drivers aged 20 to $67, M d n=40.5$, driving experience $1-40$ years, $M d n=15$. The procedure of specially designed SelfConcept IAT to measure implicit attitudes towards speeding, driver questionnaire to measure explicit attitudes towards speeding, Cross-cultural shortened form of ZKPQ-50-CC. Contexts were two videos with negative and positive emotional valence. During the first session, participants performed the IAT procedure, next completed the questionnaire. After that, the participants were divided into two groups; watching different content videos. Retest was conducted with one-week delay. Context effects led to a change in both implicit and explicit attitudes. The effect sizes of changes were typically small for implicit attitudes and medium for explicit ones. The context effect revealed a change in the correspondence of the measurement results - the appearance of inconsistency after the negative video and partial consistency-inconsistency after the positive video. Artificially created contextual events affected the system of correlation links between implicit and explicit attitudes towards speeding and personal traits.
\end{abstract}

Keywords: attitudes towards speeding, self-concept IAT, context, implicit-explicit discrepancy.

\section{Introduction}

In recent decades, theorists of implicit social cognition have paid great attention to the study of the effect of various contextual factors, both on the results of implicit measurements and on the change in implicit attitudes. A new understanding of implicit attitudes stems from an increasing amount of empirical evidence of the differences between implicit and explicit attitudes due to their differing sensitivity to context effects [1-6].

Contextual effects on implicit attitudes are associated with various sources of information, such as autobiographical real events involving self-involvement [7] and passive observation of events or stories, for example, when watching videos with an episode relevant to implicit attitude, as well as reading and imagination of events and stories [8-10].

A growing body of experimental evidence indicated that manipulation of context factors involved not only explicit but also implicit attitudes. This required a theoretical rethinking of the nature of implicit attitudes as resistant to changes in structures. The focus of attention was realized not only on the automatic nature of implicit attitudes, which was typical for the initial stage of their study and conceptualization, but also taking into account the effect of recent and past experience, affective experiences and other factors on them [11].

Since objects of attitude, including those containing new information, exist in different contextual environments, it is necessary to study how this information is integrated with the previously obtained one, and how implicit measurements reflect it [5]. The study [12] gave evidence of the contextual independence of the initially formed associations in the case of homogeneity of available information about the object of the attitude. The results of implicit measurements of attitudes became contextdependent and could be observed only in the context of contextual conditions in which conflicting information was received. Any experimental measurement procedure is based on specific cognitive mechanisms initiated during its execution.

The specifics of the measurement procedure, as well as the strategies for performing the experiment task are factors contributing to the appearance of contextual effects in implicit measurements. Due to the fact that different procedures of implicit measurements can evaluate the same thing, based on different cognitive mechanisms, experimental manipulations of contextual factors when performing 
tasks in alternative ways theoretically make it possible to determine at what level, procedural or basic, there are changes in the implicit attitude-relevant associations. Thus, the use of different procedures for implicit measurements of the same attitude under an identical experimental exposure can lead to the same or different results. If the similarity of the results of implicit measurements is obtained and there is reason to assume different cognitive mechanisms involved in the execution of the measurement procedures, then the discovered effects of the context are probably associated with changes in basic associations, or, otherwise, are observed at the procedural level [5].

Explicit attitudes have been understood as a product of primarily propositional, in fact logical processes, and implicit - mainly as a result of associative. The propositional processes are deliberate, conscious, slow, while the associative processes are fast, automatic, unconscious. Implicit measurements are a reflection of mental associations formed in the early stages of gaining social experience [13-14].

Despite the fact that there are numerous studies of implicit attitudes in social psychology and its applied fields, as well as methods of measuring them, the field of traffic psychology remains underexplored. According to the review [15], research in this area is carried out in several directions: the role of implicit and explicit attitudes that determine the driver's behavior on the road; types of measurements associated with access to implicit attitude; the connection between implicit and explicit attitudes; malleability of implicit attitudes. For example, there are studies devoted to implicit evaluations about driving skills predicting driving performance [16], examination of different predictors of different risky driving behaviors in young drivers [17], study of attitudes towards the use of mobile phones [18], implicit non- self-report measure of attitudes to speeding: development and validation [19-20].

There are only a few papers devoted to the study of the effect of contextual factors on attitudes to driving using an implicit methodology.

Sibley and Harré conducted a study of the effect of positive and negative advertising on the explicit and implicit self-perception of drivers of the ability to drive carefully [21]. Positive advertising was most effective in reducing the driver's self-confidence that he was a good driver. Negative advertising has proven to be less effective. The effect of both positive and negative advertising extended only to explicit attitudes. The effect of changing implicit attitudes was not revealed. Very brief advertisements were used that did not take into account that automatic associations are the result of slower learning processes [15]. Therefore, implicit cognitions must be resistant to short-term persuasive messages [22].

A later study [23] observed changes in implicit attitudes. In this study, a strategy of beliefs was used that appeared to be conducive to possible changes in implicit attitudes.

A review by Tosi and colleagues [15] analyzed numerous studies that showed that implicit and explicit attitudes in transportation research are weakly correlated, and that there is insufficient information about the conditions that moderate their relationship. Therefore, the question of the effects of contextual factors on the correspondence between implicit and explicit attitudes is of interest. We find a theoretical explanation of discrepancies and consistencies between implicit and explicit attitudes in modern two-process models of social cognition [24]. Despite the fact that there are differences in the mechanisms for understanding discrepancies and consistencies, there is a general assumption "that relations between measures of any kind should increase as a function of their similarity in terms of (1) the contextual conditions during the measurement process (e.g., time pressure) and (2) the mental processes involved in the production of the measured responses" [25, p. S6].

The above analysis has shown that the contextual effects on explicit and implicit attitudes towards observing the speed limit of driving have been insufficiently studied. There is a shortage of experimental implicit procedures that measure attitudes towards various forms of driving; therefore, it became necessary to create an implicit associative test (IAT) procedure to address the issue of this research.

The aim of the research is to study the context effects of graded affective valence videos on the results of measurements of implicit attitudes towards risky driving.

Research questions. Have the results of measurements of implicit attitudes towards speeding using IAT and the results of measurements of explicit attitudes using self-reported procedures changed in response to context effects? What effect did the context factors have on results consistency of implicit and explicit measurements of attitudes towards speeding? Have the correlations between implicit and 
explicit attitudes towards speeding and personality traits (Activity, Sociability, Aggression-Hostility, Impulsive Sensations Seeking, Neuroticism-Anxiety) changed in response to contextual effects?

The answers to these questions would help reveal the effect of contextual factors.

\section{Materials and methods}

Participants. 66 drivers (37 males, 29 females) with B driving licenses, aged 20-67 (Mdn= 40.5); driving experience 1-40 years $(M d n=15.0)$. Group 1: 33 participants (18 males, 15 females) whom the positive video was shown. Group 2: 33 participants (19 males, 14 females) to whom the negative video was shown.

Implicit measurements. Two-category Implicit Association Test (Self-Concept IAT) developed on the basis of Greenwald and Farnham [26]. Self-Concept IAT measures automatic associations between categories: "Self" (I, my, me, mine, self) and "Others" (others, they, them, their, it) and attributes characterizing speeding and observing speed limits.

Explicit measurements. (1) Self-assessment procedure for measuring attitudes towards speeding developed on the basis of [27]; (2) self-reported procedure Zuckerman-Kuhlman Personality Questionnaire Cross-Cultural Shortened Form (ZKPQ-50-CC) [28].

Procedure. Voluntary participation. Quasi-experimental before-after study design. During the first session, participants first performed the Self-Concept IAT procedure, next completed the questionnaire (1). After that, the participants were divided into two groups, one group watched the video clip with positive emotional valence (quiet safe driving), and the other - with the negative one (violation of the speed limit with subsequent accidents). In a week, the second measurement session was conducted. Participants performed the Self-Concept IAT procedure and completed questionnaires (1) and (2).

Self-Concept IAT experimental procedure. According to Greenwald and Farnham [26], the target category "Self" is represented by pronouns: I, my, me, mine, self; the alternative category "Others" is represented by pronouns: others, them, their, they, it. Target attributes are presented in the form of pictures associated with "Observance of speed" and "Overspeed" (Fig. 1). The stages of the IAT procedure are presented in Table 1. For each stage, the participants were presented with instructions.

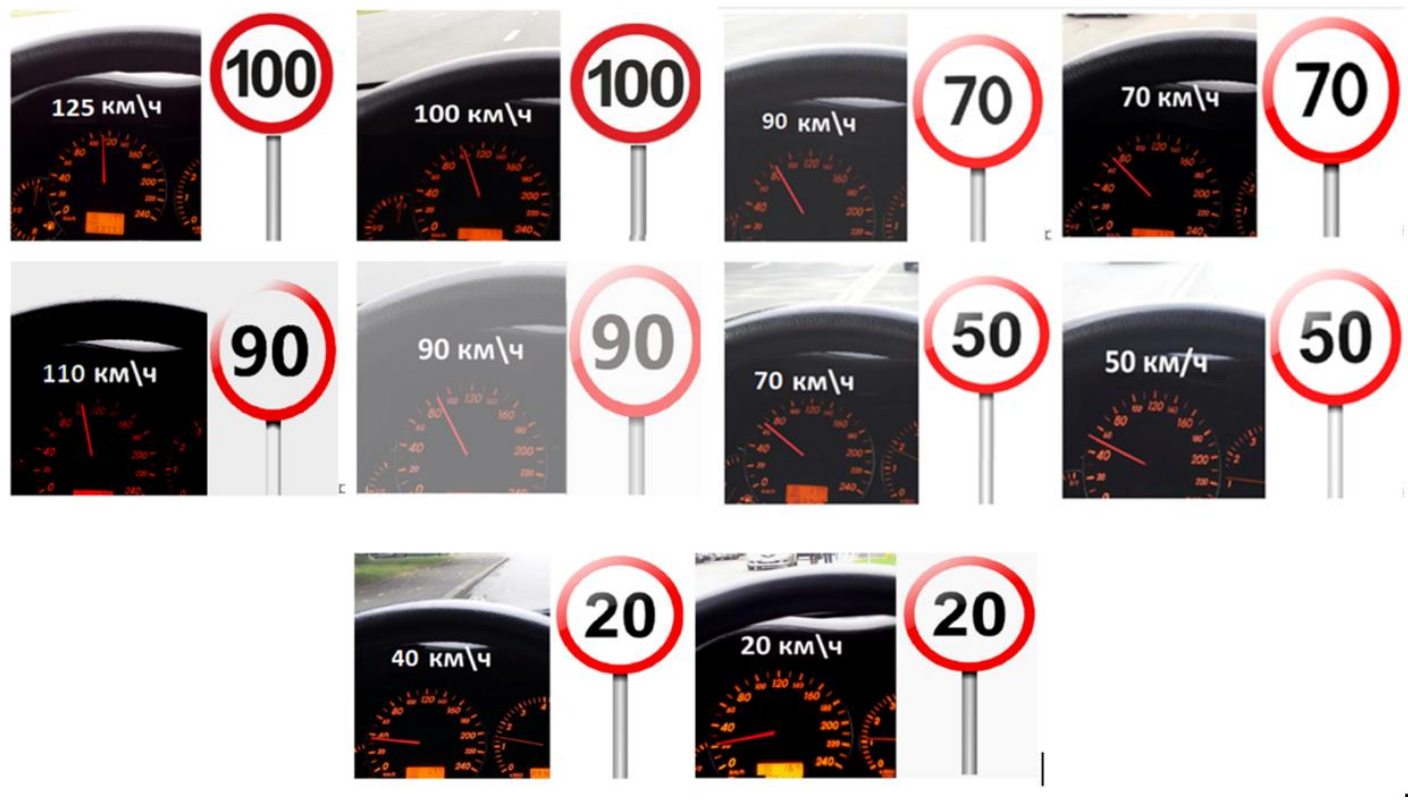

Fig. 1. Attributes related to speed observance and overspeed

The instructions were given to participants for each stage.

To calculate the effect of implicit associations, $D$-statistics [29] were used: $D$ (before), $D$ (after) before and after watching the video. At $D \leq-0.15$ - "speeding"; at $D \geq 0.15$ - "observing speed limits"; at $-0.15<D<0.15-$ implicit associations are either unformed, or unexpressed, or conflicting. In this case, we will use the notation "no preference". 
Context. Two videos of positive and negative valence were created, 1 minute and 29 seconds long each. The positive video showed the safe movement of the vehicle at the permitted speed. The negative video showed emergency situations related to violation of the speed limit, which led to accidents.

Table 1

Self-Concept IAT Procedure Stages

\begin{tabular}{|c|c|c|c|}
\hline Block & Test & Function & Procedure \\
\hline 1 & 24 & Training block & "Others" vs "Self" \\
\hline 2 & 24 & Training block & "Speeding" vs "Observing speed \\
limits"
\end{tabular}

\section{Self-reporting procedures}

Driver Questionnaire. The first and second parts of the questionnaire are developed based on the Driver Questionnaire [27]. The first part includes a number of variables theoretically related to speeding (gender, age), experience, driving - frequency of car use, odometer annual mileage (Mileage per year, $\mathrm{km}$ ), number of accidents related (and not related) to speeding (Crashes) and the number of violations of the speed limit registered by the traffic police, the use of a mobile phone while driving (Usage of mobile phone). The second part presents the Driver Behavior Scale (defines the Driver Behavior variable), which includes questions related to driving in different speed zones (for example, How often do you drive at a speed in the $50 \mathrm{~km} \cdot \mathrm{h}^{-1}$ speed zone: a) $60-65 \mathrm{~km} \cdot \mathrm{h}^{-1}$; b) more than $65 \mathrm{~km} \cdot \mathrm{h}^{-1}$ ). Answer options are represented by a 5-point Likert-type scale, from 5 - "always" to 1 - "never". High scores correspond to a violation of the speed limit. Cronbach's $\alpha=.93$. (Speeding by the Driver variable). The third part of the questionnaire, the Attitude Scale towards speeding was developed on the basis of the Driver Attitude Questionnaire (DAQ) [30]. The scale consists of 11 semantic differential scales, the poles of which are represented by words with positive and negative valence. Participants in the study need to express their assessment by answering a question like "In your opinion, speeding is bad ... - ... good", marking the appropriate gradation on a 7-step bipolar scale (from 1 - negative pole "bad" to 7 positive pole "good"). High scores on this scale indicate an attitude towards speeding. Cronbach's $\alpha=0.94$. This scale was used to measure the Attitude (before), Attitude (after) variables - explicit attitudes before and after watching the video. With Attitude $<Q_{1}-$ observing speed limits; with Attitude $>Q_{3}$ - attitude towards speeding, with $Q_{1} \leq$ Attitude $\leq Q_{3}$ the attitude is either conflicting or unformed: "no preference".

The questionnaire of personal traits "ZKPQ-50-CC" [28], Cronbach's Alpha is $\alpha=.80$. It has 50 items (direct and inverse), characterizing a person's behavior in typical life situations in which his/her personality traits are most pronounced. The scales: Impulsive-Sensation Seeking, $\alpha=0.75$; Neuroticism-Anxiety, $\alpha=0.80$; Aggression-Hostility, $\alpha=0.62$; Activity, $\alpha=0.85$; Sociability, $\alpha=0.72$. High scores indicate the severity of personality traits contained in the titles of the scales.

Apparatus: E-Prime 2.0 Professional ${ }^{\circledR}$.

\section{Results and discussion}

Variables: Session (before, after), Context (negative, positive), Gender (female, male); Age $(M d n=40.5)$; Experience ( $M d n=15$ years); Using a mobile phone while driving.

Explicit attitudes (interval variables): Attitude(before), Attitude(after) - high scores (Attitude $>Q_{3}=32$ ) correspond to attitude towards "speeding", low scores (Attitude $<Q_{1}=21$ ) correspond to attitude towards "observing speed limits"; $Q_{1} \leq$ Attitude $\leq Q_{3}$ correspond to the absence of the effect of explicit preferences: "no preference".

Explicit attitudes (nominal variables): Attitude(before), Attitude(after) with three categories of the number of participants with explicit attitudes - towards "speeding", towards "observing speed limits", the absence of the effect of explicit preferences: "no preference". 
IAT's D-scores (interval variables): $D$ (before), $D$ (after) - low scores correspond to speeding.

IAT's D-scores nominal variables): $D$ (before), $D$ (after) with three categories of the number of participants with implicit attitudes - towards "speeding", towards "observing speed limits", the absence of the effect of explicit preferences: "no preference".

Personality traits: Activity, Aggression-Hostility, Sociability, Impulsive sensation seeking, Neuroticism-Anxiety - high scores indicate the severity of personality traits contained in the titles of the scales.

To answer the first research question: "Have the results of measurements of implicit attitudes towards speeding using IAT and the results of measurements of explicit attitudes using self-reported procedures changed in response to context effects?" the Paired-Samples t-test; the frequencies analysis: Goodness-of-Fit $\left(\chi^{2}\right)$ Test, and the $\varphi^{*}$-Fisher's Angular Transformation Test; the effect sizes Cohen's $d$, Cohen's $h$ and $\chi$ were used.

Changes in the mean values of the variable D-scores after watching videos - the results of measurements of implicit attitudes are presented in Table 2. Changes in the mean values of the variable Attitude - the results of measurements of explicit attitudes are presented in Table 3. Changes in the percentage of participants with different results of measurements of implicit and explicit attitudes to speed are presented in Tables 4-5 and Figure 2.

Changing in the results of measuring implicit attitudes. After the contextual effect of videos, both negative (with episodes of speeding by road users) and positive (with episodes of observance of the speed limit), changes in the severity (mean D-scores) of the results of measurements of implicit attitudes using the Self-concept IAT for the general sample have not been identified. However, for some subgroups of participants, changes were before detected using Cohen's d effect sizes. In the group in which the negative video was shown, small, statistically insignificant changes in the results of measuring implicit attitudes were found only in the direction of observing the speed limits for the following subsets of participants: male; participants over 40.5 years old; participants with more than 15 years of driving experience; participants using a mobile phone while driving; participants with high activity; participants with high communication skills and participants with low aggressiveness-hostility (Table 2).

Table 2

Changes in average $D$-scores after showing videos. Results of Paired-Samples $t$-test

\begin{tabular}{|c|c|c|c|c|c|c|c|c|}
\hline 茪 & Subsets of participants & $N$ & 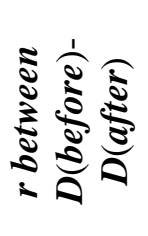 & 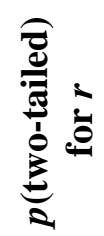 & $t$ & 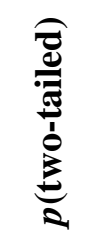 & 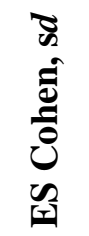 & 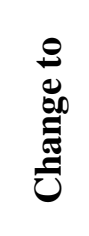 \\
\hline \multirow{7}{*}{ : } & Males & 19 & 0.49 & 0.035 & -1.07 & 0.30 & 0.25 & \multirow{7}{*}{ 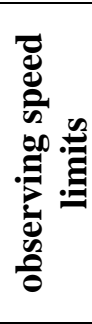 } \\
\hline & Age above 40.5 years old & 15 & 0.47 & 0.075 & -0.96 & 0.35 & 0.25 & \\
\hline & Experience above 15 years & 15 & 0.45 & 0.090 & -.914 & 0.38 & 0.24 & \\
\hline & Use the mobile phone & 16 & 0.25 & 0.35 & -0.93 & 0.37 & 0.23 & \\
\hline & Activity high & 19 & 0.33 & 0.17 & -0.81 & 0.43 & 0.19 & \\
\hline & Sociability high & 17 & 0.24 & 0.35 & -1.17 & 0.26 & 0.28 & \\
\hline & Aggression-Hostility low & 21 & 0.28 & 0.22 & -1.00 & 0.33 & 0.22 & \\
\hline
\end{tabular}

Note. Effect size Cohen's $d$ : 0.20 - small, 0.50 - medium, 0.80 - large.

Effect size $r$ : 0.10 - small, 0.30 - medium, 0.50 - large.

However, for the entire group, after showing the negative video, the percentage of participants with implicit attitude towards speeding decreased statistically insignificantly from $12 \%$ to $3 \%$. Cohen's $h$ is small, near to medium.

In the group in which the positive video was shown, small, statistically insignificant changes in the results of measuring implicit attitudes were found towards observing speed limits (for subsets of participants: under 40.5 years old; with driving experience less than 15 years; for participants with low aggressiveness-hostility and for participants with unexpressed impulsive sensation seeking) and towards speeding (for participants with more than 15 years of experience and for participants with low 
neuroticism-anxiety). It is possible that these rather experienced and calm participants were already potentially ready to grudgingly accept the attitude violation of order and monotony while driving.

After showing the positive video in the entire group, the percentage of participants with implicit attitude towards observing speed limits increased statistically insignificantly from $70 \%$ to $82 \%$. This was due to a statically insignificant reduction of the part of participants with no implicit preference (from $24 \%$ to $12 \%$ ). Effect sizes Cohen's h are between small and medium.

Both before and after the video was shown (both positive and negative), the largest percentage were participants who had an implicit preference for speed control, and the smallest percentage were participants with an implicit preference for speed. The effect size is large.

Changing the results of measuring explicit attitudes. After showing both negative and positive videos, statistically significant changes in the severity (mean values of the Attitude variable) of the results of measurements of explicit attitudes towards observing the high-speed mode of movement occurred in both groups. Effect size is between small and medium. No changes in the results of measurements of explicit attitudes were detected only in three subgroups of participants, who were shown the positive video (the group of participants under 40.5 years old, the group of participants with high activity and the group of participants with low neuroticism-anxiety), and in one subgroup of participants (with low communication skills), who were shown the negative video. The revealed changes in the results of measuring explicit attitudes in different subgroups of participants were either statistically significant, or with a trend towards statistical significance, or statistically insignificant. Effect size varied from small to large depending on the subgroup (Table 3).

After watching the negative video, the percentage of participants with explicit preference for speeding statistically insignificantly decreased from $39 \%$ to $24 \%$ (Table 4, Fig. 2).

Before the negative video was shown (before), the largest proportion were both explicit preferences for speeding (39\%) and no explicit preferences (39\%) compared to the percentage of implicit preferences for speeding (21\%). After the negative video was shown, the largest became the percentage of participants with no explicit preference (48\%), which is statistically significantly higher than the percentage of participants with explicit preference for speeding $(24 \%) .\left(\varphi^{*}=2.06, p=0.040, h=0.51\right)$, effect size Cohen's $\mathrm{h}$ is medium. It also tends to be statistically significantly higher than the percentage of participants with the explicit preference for speed observance $(27 \%)\left(\varphi^{*}=1.78, p=0.075, h=0.44\right)$, effect size Cohen's h is near to medium (Fig. 2).

After watching the positive video, the percentage of participants with explicit preference for observing the speed limit increased from $21 \%$ to $33 \%$. Also, the percentage of participants "without explicit preferences" decreased from 58\% to 45\%. In both cases, the changes are statistically insignificant, and the effect sizes are small (Table 4, Figure 2).

Before the positive video was shown, the largest proportion were participants with no explicit preferences $(58 \%)$, which statistically significantly exceeds the equal percentage of participants with explicit preference for observing the speed limit $(21 \%)$ and speeding $(21 \%)\left(\varphi^{*}=3.17, p=0.002\right.$, $h=0.78$ ), effect size Cohen's h is large.

After the positive video was shown, the largest share of the participants was also with the absence of explicit preferences (45\%), which decreased. This share is statistically significantly higher than the percentage of participants with explicit preference for speeding $(21 \%)\left(\varphi^{*}=2.11, p=0.035, h=0.52\right)$, effect size Cohen's $h$ is medium. It also statistically insignificantly exceeds the percentage of participants with explicit preference for observing the speed limit (33\%): $\varphi^{*}=1.00, p=0.32, h=0.25$, effect size Cohen's h is small.

The distributions of the percentages of participants with different preferences for the types of driving speed, both before and after contextual effect, are statistically significantly different from the uniform distribution. For implicit preferences, the effect size is large. For explicit preferences and a negative video - medium. For explicit preferences and a positive video - large before exposure, and medium after exposure. That is, the distribution has become more like a uniform distribution (Table 5).

Thus, "direct" comparisons of both the severity of changes in the results of measurements of attitudes towards speed, and the results of studying the changes using frequency analysis, led to the following conclusions on the first research question. 
Changes in the average values of the Attitude variable after showing videos,

Table 3 results of Paired-Samples $t$-test

\begin{tabular}{|c|c|c|c|c|c|c|c|}
\hline Context & Subsets & & $N$ & $t$ & $d f$ & $\begin{array}{c}p(2- \\
\text { tailed })\end{array}$ & $\begin{array}{c}\text { ES Cohen's } \\
d\end{array}$ \\
\hline negative & \multirow{2}{*}{\multicolumn{2}{|c|}{ total }} & 33 & 1.97 & 32 & 0.058 & 0.34 \\
\hline positive & & & 33 & 2.05 & 32 & 0.049 & 0.36 \\
\hline negative & \multirow{4}{*}{ Gender } & \multirow{2}{*}{ male } & 19 & 1.38 & 18 & 0.18 & 0.32 \\
\hline positive & & & 18 & 1.03 & 17 & 0.32 & 0.24 \\
\hline negative & & \multirow{2}{*}{ female } & 14 & 1.78 & 13 & 0.099 & 0.48 \\
\hline positive & & & 15 & 3.23 & 14 & 0.006 & 0.83 \\
\hline negative & \multirow{4}{*}{ Age } & \multirow{2}{*}{ low } & 18 & 1.45 & 17 & 0.17 & 0.34 \\
\hline positive & & & 15 & 0.63 & 14 & 0.54 & 0.16 \\
\hline negative & & \multirow{2}{*}{ high } & 15 & 1.32 & 14 & 0.21 & 0.34 \\
\hline positive & & & 18 & 2.18 & 17 & 0.044 & 0.51 \\
\hline negative & \multirow{4}{*}{ Experience } & \multirow{2}{*}{ low } & 18 & 1.37 & 17 & 0.19 & 0.32 \\
\hline positive & & & 16 & 1.57 & 15 & 0.14 & 0.39 \\
\hline negative & & \multirow{2}{*}{ high } & 15 & 1.38 & 14 & 0.19 & 0.36 \\
\hline positive & & & 17 & 1.31 & 16 & 0.21 & 0.32 \\
\hline negative & \multirow{4}{*}{$\begin{array}{c}\text { Usage of } \\
\text { mobile phone }\end{array}$} & \multirow{2}{*}{ no } & 17 & 0.97 & 16 & 0.34 & 0.24 \\
\hline positive & & & 19 & 1.85 & 18 & $\mathbf{0 . 0 8 0}$ & 0.42 \\
\hline negative & & \multirow{2}{*}{ yes } & 16 & 1.73 & 15 & 0.10 & 0.43 \\
\hline positive & & & 14 & 0.91 & 13 & 0.38 & 0.24 \\
\hline negative & \multirow{4}{*}{ Activity } & \multirow{2}{*}{ low } & 14 & 0.75 & 13 & 0.46 & 0.20 \\
\hline positive & & & 18 & 2.16 & 17 & 0.046 & 0.51 \\
\hline negative & & \multirow{2}{*}{ high } & 19 & 1.88 & 18 & 0.076 & 0.43 \\
\hline positive & & & 15 & 0.27 & 14 & 0.794 & 0.069 \\
\hline negative & \multirow{4}{*}{ Sociability } & \multirow{2}{*}{ low } & 16 & 0.57 & 15 & 0.58 & 0.14 \\
\hline positive & & & 17 & 1.93 & 16 & 0.072 & 0.47 \\
\hline negative & & \multirow{2}{*}{ high } & 17 & 2.13 & 16 & 0.049 & 0.52 \\
\hline positive & & & 16 & 0.86 & 15 & 0.40 & 0.22 \\
\hline negative & \multirow{4}{*}{$\begin{array}{c}\text { Impulsive } \\
\text { Sensation Seeking }\end{array}$} & \multirow{2}{*}{ low } & 19 & 1.07 & 18 & 0.30 & 0.24 \\
\hline positive & & & 15 & 1.81 & 14 & 0.091 & 0.47 \\
\hline negative & & hich & 14 & 2.03 & 13 & 0.063 & 0.54 \\
\hline positive & & लान & 18 & 1.03 & 17 & 0.32 & 0.24 \\
\hline negative & \multirow{4}{*}{ Aggression-Hostility } & \multirow{2}{*}{ low } & 21 & 1.44 & 20 & 0.17 & 0.31 \\
\hline positive & & & 15 & 1.86 & 14 & $\mathbf{0 . 0 8 5}$ & 0.48 \\
\hline negative & & \multirow{2}{*}{ high } & 12 & 1.43 & 11 & 0.18 & 0.41 \\
\hline positive & & & 18 & 1.49 & 17 & 0.15 & 0.35 \\
\hline negative & \multirow{4}{*}{ Neuroticism-Anxiety } & \multirow{2}{*}{ low } & 18 & 1.77 & 17 & 0.095 & 0.42 \\
\hline positive & & & 12 & 0.54 & 11 & 0.60 & 0.16 \\
\hline negative & & high & 15 & 0.88 & 14 & 0.39 & 0.23 \\
\hline positive & & WEII & 21 & 2.00 & 20 & 0.059 & 0.44 \\
\hline
\end{tabular}

Note. The cases without changes are marked with gray. The cases with $p<0.10$ are marked with bold. Effect size Cohen's $h$ : 0.20 - small, 0.50 - medium, 0.80 - large. 
Percentage of participants with measurement results of implicit and explicit speeding related cognitions before and after the context effect, $\varphi^{*}$-Fisher's Angular Transformation Test

\begin{tabular}{|c|c|c|c|c|}
\hline Context & Measurement Results & before & after & $\begin{array}{l}\text { Statistical Significance and } \\
\text { Effect Size }\end{array}$ \\
\hline \multirow{2}{*}{ 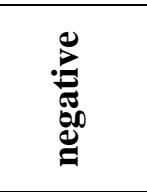 } & implicit speeding preferences & $12 \%$ & $3 \%$ & $\begin{array}{c}\varphi^{*}=1.47, p=0.14, \mathrm{~ns}, \\
h=0.36\end{array}$ \\
\hline & explicit speeding preferences & $39 \%$ & $24 \%$ & $\begin{array}{c}\varphi^{*}=1.33, p=0.18, \mathrm{~ns}, \\
h=0.33\end{array}$ \\
\hline \multirow{4}{*}{ 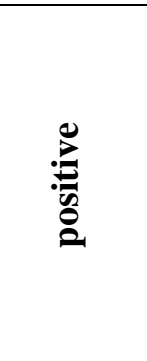 } & $\begin{array}{l}\text { explicit preferences to } \\
\text { observing the speed limit }\end{array}$ & $21 \%$ & $33 \%$ & $\begin{array}{c}\varphi^{*}=1.11, p=0.27, \mathrm{~ns}, \\
h=0.27\end{array}$ \\
\hline & no explicit preference & $58 \%$ & $45 \%$ & $\begin{array}{c}\varphi^{*}=0.99, p=0.32, \mathrm{~ns} \\
h=0.24\end{array}$ \\
\hline & $\begin{array}{l}\text { implicit preferences for } \\
\text { observing the speed limit }\end{array}$ & $70 \%$ & $82 \%$ & $\begin{array}{c}\varphi^{*}=1.16, p=0.25, \mathrm{~ns}, \\
h=0.28\end{array}$ \\
\hline & no implicit preference & $24 \%$ & $12 \%$ & $\begin{array}{c}\varphi^{*}=1.29, p=0.20, \mathrm{~ns} \\
h=0.32\end{array}$ \\
\hline
\end{tabular}

Note. Effect size Cohen's $h$ : 0.20 - small, 0.50 - medium, 0.80 - large.

Table 5

Percentage of participants with measurement results of implicit and explicit speeding related cognitions before and after the context effect. $\varphi^{*}$-Fisher's Angular Transformation Test

\begin{tabular}{|c|c|c|c|c|c|c|c|}
\hline \multirow{2}{*}{ 这 } & \multirow{2}{*}{ 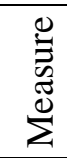 } & \multicolumn{2}{|c|}{ Difference from Uniform Distribution } & \multicolumn{2}{|c|}{$\begin{array}{l}\text { Largest share of } \\
\text { participants }\end{array}$} & \multicolumn{2}{|c|}{$\begin{array}{l}\text { Smallest share } \\
\text { of participants }\end{array}$} \\
\hline & & before & after & before & after & before & after \\
\hline \multirow{2}{*}{ 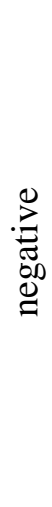 } & 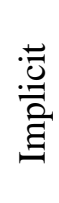 & $\begin{array}{c}\chi^{2}(2, N=33)=23.1, \\
p<0.001, \chi=0.84\end{array}$ & $\begin{array}{c}\chi^{2}(2, N=33)=28.4, \\
p<0.001, \chi=0.93\end{array}$ & 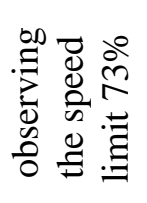 & 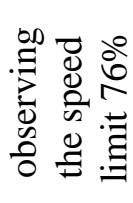 & 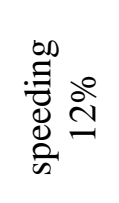 & 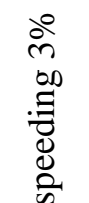 \\
\hline & 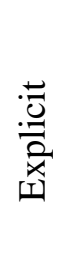 & $\begin{array}{c}\chi 2(2, N=33)=2.18 \\
p=0.34, \chi=0.26\end{array}$ & $\begin{array}{c}\chi^{2}(2, N=33)=3.46 \\
p=0.18, \chi=0.32\end{array}$ & 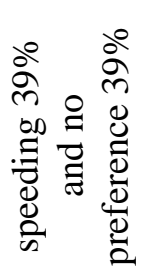 & 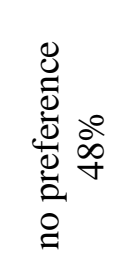 & 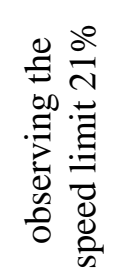 & 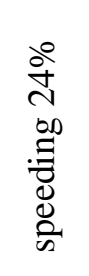 \\
\hline \multirow{2}{*}{$:$} & 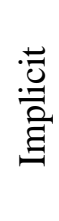 & $\begin{array}{c}\chi^{2}(2, N=33)=21.3 \\
p<0.001, \chi=0.80\end{array}$ & $\begin{array}{c}\chi^{2}(2, N=33)=35.1, \\
p<0.001, \chi=1.03\end{array}$ & 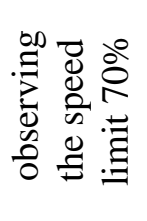 & 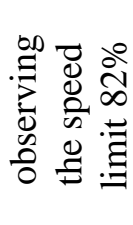 & $\begin{array}{l}0 \\
0 \\
o 0 \\
: \\
0 \\
0 \\
0 \\
0\end{array}$ & 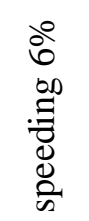 \\
\hline & 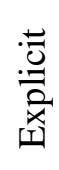 & $\begin{array}{c}\chi^{2}(2, N=33)=8.73 \\
p=0.013, \chi=0.51\end{array}$ & $\begin{array}{c}\chi^{2}(2, N=33)=2.91 \\
p=0.23, \chi=0.30\end{array}$ & 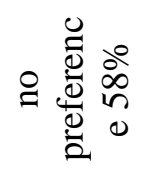 & 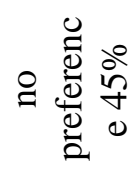 & 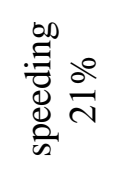 & 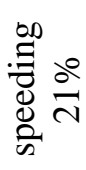 \\
\hline
\end{tabular}

Note. Effect size $\chi: 0.10$ - small, 0.30 - medium, 0.50 - large.

First, watching the video had a more pronounced effect on changes in the results of measuring explicit attitudes than on changes in the results of measuring implicit attitudes, both in terms of statistical significance and in terms of the size of the effects of changes. This result is partially consistent with the result of the study $[21 ; 31]$. 
Secondly, the revealed changes in the measurement results of both implicit and explicit attitudes were directed towards observing the speed limit when driving. This fact can be used to develop and implement similar contextual factors in practical work with drivers.

Thirdly, the results obtained confirm the well-known scientific theses on the greater sensitivity of explicit attitudes to contextual effects in comparison with implicit ones [32-34].

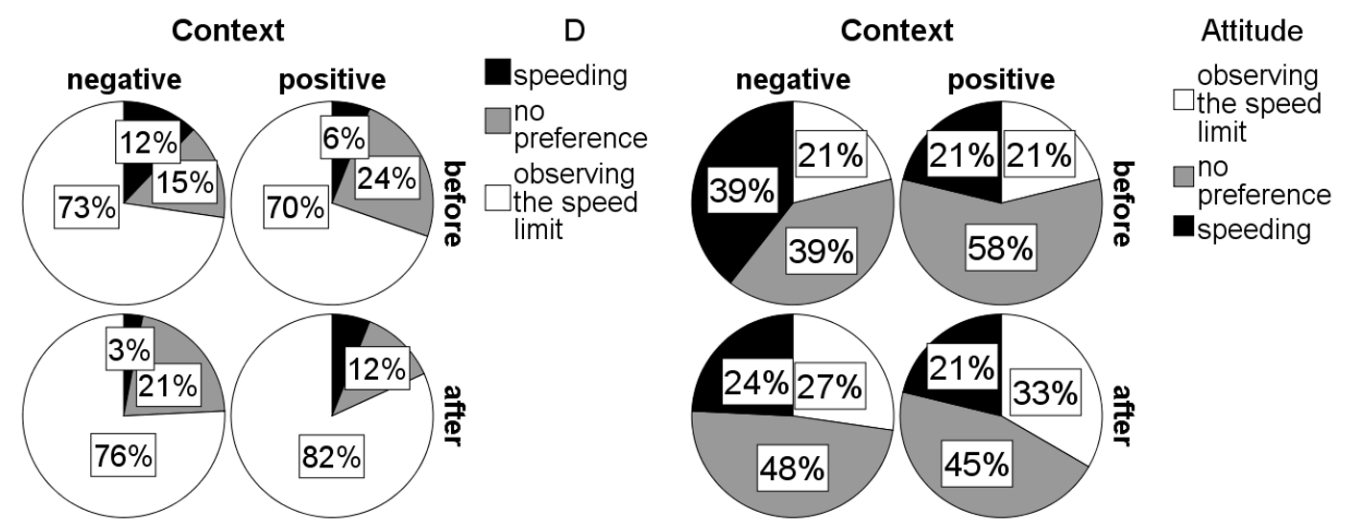

Fig. 2. Percentage of the participants with measurement results of implicit and explicit speeding related cognitions before and after the context effect

To answer the second research question: "What effect did the context factors have on results consistency of implicit and explicit measurements of attitudes towards speeding?" the correlation analysis was used. Effect sizes $r(0.10$ - small, 0.30 - medium, $0.50-$ large $)$.

Consistency of the measurement results is understood as the correspondence of the measurement results to implicit and explicit attitudes towards speed. In this research, the correspondence between the results of implicit and explicit measurements is carried out using the correlation coefficients. According to the definition of the D-scores variables for measuring implicit attitudes and the Attitude variable for measuring explicit attitudes (see the beginning of the Results and Discussion section), if the measured results match the correlation coefficients must be negative.

Inconsistency is understood as a divergence between the results of measurements of implicit and explicit attitudes towards speed. In this research, with inconsistency, the correlation coefficients should be positive.

Uncertain consistency is understood as the absence of both consistency, and inconsistency (the absolute value of the correlation coefficients does not exceed 0.10 ).

Correlation coefficients indicating the consistency, inconsistency and uncertain consistency of the results of measurements of implicit and explicit attitudes before and after showing videos for two groups and their subgroups are shown in Table 6.

Fig. 3 depicts the scatters with linear regression lines for the group in which the negative video was shown and for the group in which the positive video was shown. The vertical axis shows the values of the $D$-scores variable, and the horizontal axis shows the values of the Attitude variable. The black points and black regression line correspond to measurements before exposure. Gray dots and gray regression line correspond to post-exposure measurements. After exposure to the negative roller, the gray regression line has the largest slope and makes an acute angle with the positive direction of the horizontal axis. This indicates a positive correlation, that is, the inconsistency of the measurement results. As it can be seen from Table 6, the uncertain consistency of the measurement results was observed prior to the negative roller exposure. After its impact, a positive statistically significant correlation coefficient is recorded $r(33)=0.36, p=0.041$, the effect size is above medium. This indicates the presence of inconsistency in the measurement results.

Before showing the negative video, the consistency of the results of implicit and explicit measurements was observed in the subgroups, such as: Females, Experience up to 15 years, Aged up to 40.5, Not using mobile phone while driving, Low Activity, Low Sociability, High Impulsive Sensation Seeking, Low Neuroticism-Anxiety. After showing the negative video, in all, without exception, subgroups there was inconsistency of the results of implicit and explicit measurements. 
In the other group before the display of the positive video, there was a very weak consistency effect: $r(33)=-0.11, p=0.56$, ns. After the display of the video, there was an uncertain consistency: $r(33)=0.070, p=0.70$.

Before showing the positive video, the consistency of the results of implicit and explicit measurements was observed in the subgroups: Females, Experience above 15 years, Aged above 40.5, Using mobile phone while driving, Low Activity, High Sociability, Low and high Aggression-Hostility, Low and high Impulsive Sensation Seeking, High Neuroticism-Anxiety. After showing the positive video the consistency was observed in the subgroups, such as: Females, Low Activity, Low Sociability. Inconsistency was observed in the subgroups, such as: Males, Experience up to 15 years, Aged below 40.5, High Activity, High Sociability, Low Neuroticism-Anxiety.

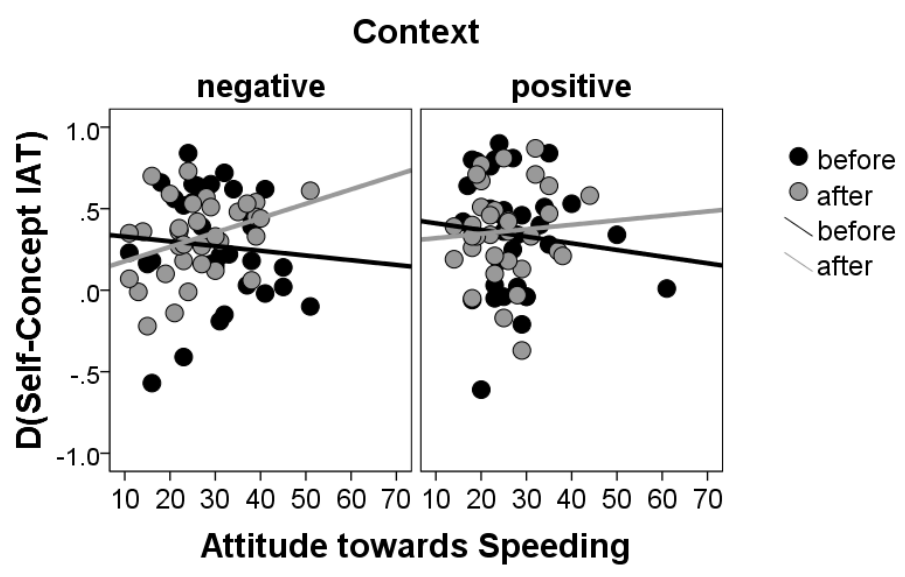

Fig. 3. Scatter with the linear regression lines. D-scores - Attitude variables. The black points and the black line correspond to the measurements before the context effect, the gray points and the gray line - after the context effect

As noted by Gawronski [25], dissociations between implicit and explicit measures are manifested in three forms: they are rather weakly correlated; predict different types of behavior or the same behavior under different conditions; differ in their sensitivity to the same external exposure.

For example, according to the APE model, implicit and explicit attitude measures should be discrepant when information automatically activated from memory is inconsistent with propositional information. The truth value (or validity) of a propositional inference determines whether there are discrepancies [33-34].

It is possible that as a result of watching the negative video, there was either a change in the information on which the associations are based, or (most likely) in the information on which the propositional is based, which could lead to inconsistency of the results of implicit and explicit measurements of attitudes towards speeding.

In our research, at the demonstration of the negative video, most likely, the explicit measures were more sensitive to its impact.

It is possible that the results of the analysis of the second research question will somehow complement the analysis of numerous studies from the review by Tosi and colleagues [15], in which it was shown that implicit and explicit attitudes in transportation research are weakly correlated, and that there is not enough information about conditions that moderate their relationship.

The results obtained in this research confirmed the assumption of Fazio and Olson [35] about the existence of experimental conditions under which the consistency of the results of implicit and explicit measurements can exist. Similar results were obtained in researches [18; 36-37], where consistency in the results of implicit and explicit measurements was observed only in individual subgroups of participants. 
Consistency, inconsistency and uncertain consistency of the measurement results,

Pearson's correlation coefficients

\begin{tabular}{|c|c|c|c|c|c|c|c|c|c|c|c|c|c|}
\hline \multirow{4}{*}{ Variable } & \multirow{4}{*}{ Subset } & \multicolumn{12}{|c|}{ Context } \\
\hline & & \multicolumn{6}{|c|}{ negative } & \multicolumn{6}{|c|}{ positive } \\
\hline & & \multicolumn{3}{|c|}{ before } & \multicolumn{3}{|c|}{ after } & \multicolumn{3}{|c|}{ before } & \multicolumn{3}{|c|}{ after } \\
\hline & & $r$ & $N$ & $p$ & $r$ & $N$ & $p$ & $r$ & $N$ & $p$ & $r$ & $N$ & $p$ \\
\hline & Total & -0.084 & 33 & 0.64 & 0.36 & 33 & 0.041 & -0.11 & 33 & 0.56 & 0.070 & 33 & 0.70 \\
\hline \multirow{2}{*}{ Gender } & Female & -0.26 & 14 & 0.36 & 0.38 & 14 & 0.18 & -0.53 & 15 & 0.041 & -0.19 & 15 & 0.50 \\
\hline & Male & \begin{tabular}{|l|}
0.024 \\
\end{tabular} & 19 & 0.92 & 0.34 & 19 & 15 & 0.061 & 18 & 0.81 & 0.30 & 18 & 0.22 \\
\hline \multirow{2}{*}{$\begin{array}{c}\text { Experienc } \\
e\end{array}$} & $\begin{array}{c}\text { Before } 15 \\
\text { years }\end{array}$ & 41 & 18 & 0.095 & 0.30 & 18 & 0.23 & 0.092 & 16 & 073 & 0.18 & 16 & 0.51 \\
\hline & $\begin{array}{c}\text { Above } 15 \\
\text { years }\end{array}$ & 0.046 & 15 & 0.87 & 0.40 & 15 & 0.14 & -0.29 & 17 & .026 & -0.034 & 17 & 0.90 \\
\hline \multirow{2}{*}{ Age } & $\begin{array}{l}\text { Below } \\
M d n\end{array}$ & .40 & 18 & 0.10 & 0.32 & 18 & 0.20 & -0.059 & 15 & 0.84 & 0.12 & 15 & 0.66 \\
\hline & $\begin{array}{l}\text { Above } \\
M d n\end{array}$ & 0.028 & 15 & 0.92 & 0.40 & 15 & 0.14 & -0.14 & 18 & 0.59 & -0.030 & 18 & 0.95 \\
\hline \multirow{2}{*}{$\begin{array}{c}\text { Usage of } \\
\text { mob. } \\
\text { phone }\end{array}$} & mo & .38 & 17 & 0.14 & 0.23 & 17 & .38 & -0.056 & 19 & 0.82 & 0.025 & 19 & 0.92 \\
\hline & yes & 0.19 & 16 & 0.49 & 0.46 & 16 & 0.072 & -0.21 & 14 & 0.47 & 0.076 & 14 & 0.80 \\
\hline \multirow{2}{*}{ Activity } & low & \begin{tabular}{|l|}
-0.23 \\
\end{tabular} & 14 & 0.43 & 0.54 & 14 & 0.047 & -0.36 & 18 & 0.14 & -0.21 & 18 & 0.41 \\
\hline & high & \begin{tabular}{|l|}
0.011 \\
\end{tabular} & 19 & 0.97 & 0.17 & 19 & 0.50 & 0.11 & 15 & 0.69 & 0.29 & 15 & 0.30 \\
\hline \multirow{2}{*}{ Sociability } & low & -0.33 & 16 & 0.21 & 0.41 & 16 & 0.12 & -0.061 & 17 & 0.82 & -0.18 & 17 & 0.49 \\
\hline & high & \begin{tabular}{|l|l|}
0.029 \\
\end{tabular} & 17 & 0.91 & 0.27 & 17 & 0.30 & -0.19 & 16 & 0.49 & 0.14 & 16 & 0.61 \\
\hline \multirow{2}{*}{$\begin{array}{l}\text { Aggressio } \\
n \text {-Hostility }\end{array}$} & low & 0.054 & 21 & 0.82 & 0.37 & 21 & 0.10 & -0.14 & 15 & 0.62 & 0.072 & 15 & 0.80 \\
\hline & high & \begin{tabular}{|c|}
-0.45 \\
\end{tabular} & 12 & 0.14 & 0.39 & 12 & 0.22 & -0.19 & 18 & 0.46 & 0.095 & 18 & 0.71 \\
\hline \multirow{2}{*}{$\begin{array}{c}\text { Impulsive } \\
\text { Sensation } \\
\text { Seeking }\end{array}$} & low & \begin{tabular}{|l|}
0.054 \\
\end{tabular} & 21 & 0.82 & 0.37 & 21 & 0.10 & -0.14 & 15 & 0.62 & 0.072 & 15 & 0.80 \\
\hline & high & -0.45 & 12 & 0.14 & 0.39 & 12 & 0.22 & -0.19 & 18 & 0.46 & 0.095 & 18 & 0.71 \\
\hline \multirow{2}{*}{$\begin{array}{l}\text { Neuroticis } \\
m \text {-Anxiety }\end{array}$} & low & \begin{tabular}{|c|}
-0.20 \\
\end{tabular} & 18 & 0.43 & 0.35 & 18 & 0.15 & 0.24 & 12 & 0.46 & 0.14 & 12 & 0.66 \\
\hline & high & 0.006 & 15 & 0.98 & 0.38 & 15 & 0.17 & -0.16 & 21 & 0.49 & 0.041 & 21 & 0.86 \\
\hline
\end{tabular}

Note. Bold - the consistency of the measurement results. Gray bold - the inconsistency of the measurement results. Effect size $r: 0.10$ - small, 0.30 - medium, 0.50 - large.

To answer the third research question: "Have the correlations between implicit and explicit attitudes towards speeding and personality traits (Activity, Sociability, Aggression-Hostility, Impulsive Sensations Seeking, Neuroticism-Anxiety) changed in response to contextual effects?" the correlation analysis was used.

Changes in the association of implicit and explicit attitudes with personality traits after showing negative or positive videos are shown in Table 7.

After the negative video was shown, the association between the explicit attitude to speed and Neuroticism-Anxiety disappeared.

After the negative video was shown, the association between the measured implicit attitude and Impulsive Sensation Seeking disappeared. The association between implicit attitudes and NeuroticismAnxiety emerged. The association between implicit attitudes and Aggression-Hostility emerged.

After showing the positive video, the association between the explicit attitude and Activity disappeared. The association between explicit attitudes and Neuroticism-Anxiety has become weaker. The association between the explicit attitude and Sociability emerged. The association between explicit attitudes and Impulsive Sensation Seeking emerged. 
Explicit attitude towards speeding before and after context,

Table 7 changes of associations with personal traits

\begin{tabular}{|c|c|c|c|}
\hline \multicolumn{4}{|c|}{ Negative video } \\
\hline Explicit attitude & before & after & Changes \\
\hline Activity & $r(33)=0.026, p=0.89$ & $r(33)=0.076, p=0.67$ & not found \\
\hline Aggression-Hostility & $\mathrm{r}(33)=0.037, \mathrm{p}=0.84$ & $\mathrm{r}(33)=0.039, \mathrm{p}=0.83$ & not found \\
\hline Sociability & $r(33)=0.026, p=0.89$ & $r(33)=-0.079, p=0.66$ & not found \\
\hline $\begin{array}{l}\text { Impulsive Sensation } \\
\text { Seeking }\end{array}$ & $r(33)=0.17, p=0.33$ & $r(33)=0.19, p=0.29$ & not found \\
\hline Neuroticism-Anxiety & $r(33)=-0.14, p=0.43$ & $r(33)=-0.087, p=0.63$ & changed \\
\hline Implicit attitude & before & after & \\
\hline Activity & $r(33)=-0.12, p=0.49$ & $r(33)=-0.14, p=0.45$ & not found \\
\hline Aggression-Hostility & $r(33)=-0.066, p=0.71$ & $r(33)=-0.15, p=0.41$ & changed \\
\hline Sociability & $r(33)=-0.32, p=0.069$ & $r(33)=-0.32, p=0.072$ & not found \\
\hline $\begin{array}{l}\text { Impulsive Sensation } \\
\text { Seeking }\end{array}$ & $r(33)=0.12, p=0.49$ & $r(33)=0.060, p=0.74$ & changed \\
\hline Neuroticism-Anxiety & $r(33)=-0.087, p=0.63$ & $r(33)=-0.17, p=0.34$ & changed \\
\hline \multicolumn{4}{|c|}{ Positive video } \\
\hline Explicit attitude & before & after & Changes \\
\hline Activity & $r(33)=-0.26, p=0.14$ & $r(33)=-0.073, p=0.69$ & changed \\
\hline Aggression-Hostility & $r(33)=0.30, p=0.085$ & $r(33)=0.27, p=0.13$ & not found \\
\hline Sociability & $r(33)=0.077, p=0.67$ & $r(33)=0.33, p=0.058$ & changed \\
\hline $\begin{array}{l}\text { Impulsive Sensation } \\
\text { Seeking }\end{array}$ & $r(33)=0.084, p=0.64$ & $r(33)=0.16, p=0.36$ & changed \\
\hline Neuroticism-Anxiety & $r(33)=0.42, p=0.016$ & $r(33)=0.15, p=0.41$ & changed \\
\hline Implicit attitude & before & after & \\
\hline Activity & $r(33)=-0.081, p=0.65$ & $r(33)=-0.080, p=0.66$ & not found \\
\hline Aggression-Hostility & $r(33)=0.13, p=0.46$ & $r(33)=0.050, p=0.78$ & changed \\
\hline Sociability & $r(33)=0.28, p=0.11$ & $r(33)=0.29, p=0.096$ & not found \\
\hline $\begin{array}{l}\text { Impulsive Sensation } \\
\text { Seeking }\end{array}$ & $r(33)=0.24, p=0.19$ & $r(33)=0.28, p=0.12$ & not found \\
\hline Neuroticism-Anxiety & $r(33)=-0.17, p=0.34$ & $r(33)=-0.041, p=0.82$ & changed \\
\hline
\end{tabular}

After the positive video was shown, the association between the implicit attitude and AggressionHostility disappeared. The association between the implicit attitude and Neuroticism-Anxiety disappeared. Thus, viewing both negative and positive videos in some cases led to a change in some associations (disappearance, appearance, and change in the strength of connection) of implicit and explicit attitudes towards the speed mode of driving with personality traits. The reasons for the changes in these associations, most likely, are the changes in the attitudes themselves.

The results of this research were presented in the form of a report at the XVI European Congress of Psychology (ECP-2019) with publication of the abstract in its proceedings [38].

The major limitation of the research is the small sample size, which, on the one hand, did not allow obtaining statistically significant results where they could be obtained, and, on the other hand, limited the possibilities of constructing an experimental design that allows considering both experimental and control groups of participants with different delays in repeated measurements after watching videos. This limitation is planned to be removed in future research. Also, in future research to measure the implicit attitudes towards the speed mode of driving, it is proposed to use the evaluative priming procedure. 


\section{Conclusions}

The present research is based on the main theoretical conceptualizations of explicit-implicit attitudes, discrepancy-consistency of explicit and implicit measurements, presented in various twoprocess attitude models.

Special attention has been paid to the theoretical understanding of the concept of context and its role in the formation and change of implicit attitudes. There is a paucity of studies examining the impact of contextual factors on driving attitudes using implicit methodology.

Reviews of traffic psychology attitudes research indicate that currently empirical studies are focused on the role of implicit attitudes in predicting driver behavior on the road, on the development of implicit tools, and special attention is paid to the issue of matching implicit and explicit dimensions of attitudes.

To implement the aim of the research, the quasi-experimental design was created, the contextual effect was developed, and the Self-Concept IAT experimental procedure was created.

The research participants performed the Self-concept IAT procedure and self-reporting procedures related to the measurement of implicit and explicit attitudes towards the driving speed. Immediately after completing these procedures, the participants were shown videos. Some - positive (measured, calm driving, observing the speed limit), others - negative (driving with excess of the speed limit, as a result of which accidents with serious consequences occur). One week after watching the videos, the research participants repeated the same measurement procedures. The results of the measurements were compared and some changes in attitudes were found, which were probably due to watching the video.

The following methods were used to determine the change in implicit and explicit attitudes towards the speed mode of driving. Before and after the context effect, we compared.

- severity of the measured attitudes;

- percentage of participants by category of their speed attitudes (overspeed, observance, lack of "preferences");

- correspondence of the results of implicit and explicit measurements;

- associations with personality traits.

Only statistically insignificant effects were found for changes in implicit attitudes towards driving speed, most of which showed a shift towards observing the speed limit, regardless of the valence of the video. More detailed studies have identified subsets of participants with more pronounced changes in implicit attitudes.

For changes in explicit attitudes towards the driving speed, it was possible to find both statistically significant and insignificant effects of large sizes and changes that also show changes in explicit attitudes towards speeding in favor of observing speed limits. No changes were found in the groups of participants: age below the median, with high activity, with low neuroticism-anxiety.

The results obtained are consistent with existing ideas about the greater sensitivity of explicit attitudes to contest effects as compared to implicit ones.

Before the negative video was shown, in some subgroups of participants, both the consistency of the results of implicit and explicit measurements and their mismatch were observed. After the negative video was shown in all subgroups without exception, there was a discrepancy between the results of implicit and explicit measurements.

Before the positive video was shown, consistency between the results of implicit and explicit measurements was observed in some subgroups of participants. After showing the positive video, the consistency of the results decreased, disappeared, or changed to inconsistency. It was not possible to establish the regularity of these changes.

Viewing both negative and positive videos in some cases led to a change in some of the associations of the implicit and explicit attitudes towards the speed mode of driving with personality traits. The reasons for the changes in these associations, most likely, are the changes in the attitudes themselves.

Thus, the aim of the research has been achieved and answers to research questions have been obtained. 


\section{Acknowledgements}

The authors are grateful to Masters of Psychology, graduates of the Baltic International Academy Jana Čužujeva, Vladislavs Sirica and Vladimirs Dementjevs for their participation in the creation of videos and for help in conducting the research.

\section{References}

[1] Gawronski B., Sritharan R. Formation, change, and contextualization of mental associations: Determinants and principles of variations in implicit measures. In B. Gawronski and B. K. Payne (Eds.), Handbook of implicit social cognition: Measurement, theory, and applications. New York: Guilford Press, 2010, pp. 216-240.

[2] Blair I. V. The malleability of automatic stereotypes and prejudice. Personality and Social Psychology Review, vol. 6, 2002, pp. 242-261. DOI: 10.1207/S15327957PSPR0603_8.

[3] Fazio R. H., Olson, M. A. Implicit measures in social cognition research: Their meaning and use. Annual Review of Psychology, vol. 54, 2003, pp. 297-327.

[4] Fazio R. H. Attitudes as object-evaluation associations of varying strength. Social Cognition, vol. 25, 2007, pp. 603-637.

[5] Plotka I., Igonin, D., Blumenau, N. Implicit Attitudes and Measurements: Effect of Context. International Business: Innovations, Psychology and Economics, vol. 7, No 2(12), 2016, pp. 7-150. [online] [21.03.2021] Available at: http://www.kuryba.lt/failai/zurnalai/2016_2.pdf.

[6] Плотка И.Д., Игонин Д.А., Блюменау Н.Ф. Оценка влияния контекстного фактора на результаты измерения имплицитных аттитюдов при разных SOA с помощью индекса имплицитного предпочтения (Assessment of effect of the contextual factor on the measurement results of implicit attitudes at various SOAs using implicit preference index). Активизация ментальных ресурсов субъекта: теория и практика Материалы IV международного симпозиума 6-7 июля 2017 г. In G.V. Ozhiganova I.D. Plotka (Eds.), Activation of mental resources of personality: Theory and practice. Proceedings of the IV International Symposium July 6-th - 7-th, 2017 (Latvia, Riga). Riga: BIA, 2017, pp. 62-73. 117 p. (In Russian).

[7] Marini M., Rubichi S., Sartori G. The role of self-involvement in shifting IAT effects. Experimental Psychology, vol. 59, No 6, 2012, pp. 348-354.

[8] Foroni F., Mayr U. The power of a story: New, automatic associations from a single reading of a short scenario. Psychonomic Bulletin and Review, vol. 12, 2005, pp. 139-144.

[9] Correll J., Park B., Judd C. M., Wittenbrink B. (2007). The effect of stereotypes on decisions to shoot. European Journal of Social Psychology, vol. 37, 2007, pp. 1102-1117.

[10] Marini M., Rubichi S., Sartori G. Implicit racial stereotypes may temporarily shift after reading a story. In L. Carlson, C. Hoelscher, T. Shipley (Eds.), Proceedings of the 33rd Annual Conferences of "Cognitive Science Society", July 20-23, 2011, Boston, Massachusetts, USA, pp. 1224-1229.

[11] Rudman L. A. Sources of Implicit Attitudes. Current Direction in Psychological Science, vol. 13, 2013, pp. 79-82.

[12] Rydell R. J., Gawronski B. I like you, I like you not: Understanding the formation of context dependent automatic attitudes. Cognition and Emotion, vol. 23, 2009, pp. 1118-1152.

[13] DeHart T., Pelham B. W., Tennen, H. What lies beneath: Parenting style and implicit self-esteem. Journal of Experimental Social Psychology, vol. 42, 2006, pp. 1-17.

[14] Rudman L. A. Sources of implicit attitudes. Current Directions in Psychological Science, vol. 13, 2004, pp. 79-82.

[15] Tosi J.D., Haworth N., Díaz-Lázaro C.M., Poó F.M., de Ledesma R. Implicit and explicit attitudes in transportation research: A literature review. Transportation Research Part F: Traffic Psychology and Behaviour, vol. 77, 2021, pp. 87-101. DOI: 10.1016/j.trf.2020.12.014.

[16] Biçaksız P., Harma M., Doğruyol B., Lajunen T., Özkan T. Implicit evaluations about driving skills predicting driving performance. Transportation research part F: Traffic psychology and behaviour, vol. 54, 2018, pp. 357-366.

[17] Fernandes R., Hatfield J., Job R. S. Factors associated with speeding, drink-driving, driving while fatigued and seat belt use in a sample of metropolitan Sydney drivers. "Australasian Road Safety Research, Policing and Education Conference", October 25-27, 2006, Sydney: [NSW Road and Traffic Authority], The Centre for Automotive Safety Research provided this page with links to the 
PDF versions of all of the papers that we were able to find for the 2006-2011 conferences. [online] [21.03.2021] Available at: http://casr.adelaide.edu.au/rsr/RSR2006/FernandesR.pdf

[18] Vinogradova Z., Plotka I., Smirnova I., Blumenau N., Igonin D. Study of attitudes towards the use of mobile phones while driving with implicit association tests and self-assessment procedures. In V. Lubkina, S. Usca, \& A. Zvaigzne (Eds.), Proceedings of the International Scientific Conference "Society. Integration. Education" May 26-27, 2018. Rezekne: Rezeknes Academy of Technologies, Latvia, vol. 7, pp. 291-307.

Available at: DOI: 10.17770/sie2018vol1.3397.

[19] Hatfield J., Fernandes R., Faunce G., Job R. S. F. An implicit non-self-report measure of attitudes to speeding: Development and validation. Accident Analysis and Prevention, vol. 40, 2008, pp. 616 - 627. http://dx.doi.org/10.1016/j.aap.2007.08.020.

[20] Martinussen L.M., Sømhovd, M.J., Møller M., Siebler F. A Go/No-go approach to uncovering implicit attitudes towards safe and risky driving. Transportation Research Part F: Traffic Psychology and Behaviour, vol. 30, 2015, pp. 74-83.

[21] Sibley C., Harré N. The impact of different styles of traffic safety advertisement on young drivers' explicit and implicit self-enhancement biases. Transportation Research Part F: Traffic Psychology and Behaviour, vol. 12, 2009, pp. 159-167.

[22] Rydell R. J., McConnell A. R. Understanding implicit and explicit attitude change: A systems of reasoning analysis. Journal of Personality and Social Psychology, vol. 91, No 6, 2006, pp. 9951008. DOI: 10.1037/0022-3514.91.6.995

[23] Manca S., Altoè G., Schultz P.W., Fornara F. The Persuasive route to sustainable mobility: elaboration likelihood model and emotions predict implicit attitudes. Environment and Behavior, vol. 52, No 8, 2019, pp. 830-860. DOI: 10.1177/0013916518820898.

[24] Rydell R.J., McConnell A.R. Consistency and inconsistency in implicit social cognition: The case of implicit and explicit measures of attitudes. In: B. Gawronski, B. K. and Payne (Eds.), Handbook of implicit social cognition: Measurement, theory, and applications. New York: Guilford Press, 2010, pp. 295-310.

[25] Gawronski B., De Houwer A.J., Sherman J.W. Twenty-five years of research using implicit measures. Social Cognition, vol. 38, 2020, pp. S1-S25.

[26] Greenwald A. G., Farnham S. D. Using the Implicit Association Test to measure self-esteem and self-concept. Journal of Personality and Social Psychology, vol. 79, 2000, pp. 1022-1038.

[27] Hatfield J., Fernandes R., Faunce G., Job R. S. F. Development of an Implicit Association Test to measure attitudes toward speeding. NSW Injury Risk Management Research Centre. ATSB research and analysis road safety research grant report 2007-05. New South Wales: University of New South Wales, 2007. 57 p. [online] [21.03.2021] Available at: https://www.infrastructure.gov.au/roads/safety/publications/2007/pdf/Granr_Report200705.pdf.

[28] Aluja A., Rossier J., Garcia L.F., Angleitner A., Kuhlman M., Zuckerman M. A cross-cultural shortened form of the ZKPQ (ZKPQ-50-cc) adapted to English, French, German, and Spanish languages. Personality and Individual Differences, vol. 41, 2006, pp. 619-628.

[29] Rudman L. A. Implicit measures for social and personality psychology. London: Sage, 2011. p.77.

[30] Conner M., Lawton R., Parker D., Chorlton K., Manstead A.S.R., Stradling S. Application of the theory of planned behavior to the prediction of objectively assessed breaking of posted speed limits. British Journal of Psychology, vol. 98, 2007, pp. 429-453.

[31] Sibley C. G., Harre N. A gender role socialization model of explicit and implicit biases in driving self-enhancement. Transport Research Part F, vol. 12, No 6, 2009, pp. 452-462.

[32] Rydell R. J., McConnell A. R., Strain, L. M. Claypool, H. M., Hugenberg K. Implicit and explicit attitudes respond differently to increasing amounts of counteranitudinal information. European Journal of Social Psychology, vol. 37, 2007, pp. 867-878.

[33] Gawronski B., Bodenhausen G. V. Associative and propositional processes in evaluation: An integrative review of implicit and explicit attitude change. Psychological Bulletin, vol. 132, 2006, pp. 692-731.

[34] Gawronski B., Bodenhausen G. V. Associative and propositional processes in evaluation: Conceptual, empirical, and meta-theoretical issues. Psychological Bulletin, vol. 132, 2006, pp. 745750 . 
[35] Fazio R. H., Olson M. A. Implicit measures in social cognition research: Their meaning and use. Annual Review of Psychology, vol. 54, 2003, pp. 297-327.

[36] Plotka I., Blumenau N., Igonin D., Bolshakova A. Research of relationships between implicit and explicit healthy or unhealthy food related cognitions. In V. Lubkina, S. Usca, \& A. Zvaigzne (Eds.), Proceedings of the International Scientific Conference "Society. Integration. Education". May 2627, 2019, Vol. I, pp.97-121. Rezekne: Rezeknes Academy of Technologies. DOI: $10.17770 /$ sie2019vol7.3888.

[37] Plotka I., Simane-Vigante L., Blumenau N. Implicit association Self-concept test in studying of violence-related cognitions. Social psychology and society, vol. 9, No 3, 2018, pp. 176-186. Available at doi:10.17759/sps.2018090317.

[38] Plotka I., Blumenau N., Igonin D., Vinogradova Z., Syritsa V. Impact of relevant episodic events on the results of implicit measurements of the attitude towards speeding. Book of Abstracts of "XVI European Congress of Psychology. (ECP-2019)", July 2-5, 2019, Lomonosov Moscow State University, Moscow, p. 1047. Moscow: University Press. [online] [21.03.2021] Available at: https://ecp2019.ru/doc/Book_of_Abstracts_ecp_2019.pdf. 